\title{
Bi-Metallic Magnetic Wire With Insulating Layer as Core for Orthogonal Fluxgate
}

\author{
M. Butta ${ }^{1}$, P. Ripka ${ }^{1}$, G. Infante ${ }^{2}$, G. A. Badini-Confalonieri ${ }^{2}$, and M. Vázquez ${ }^{2}$ \\ ${ }^{1}$ Faculty of Electrical Engineering, Department of Measurement, Czech Technical University, Prague, Czech Republic \\ ${ }^{2}$ Institute for Materials Science of Madrid, CSIC, Madrid. Spain
}

In this paper, we examine the problems related to orthogonal fluxgates realized using magnetic microwires as core. Starting from a description of orthogonal fluxgates evolution, we give a theoretical analysis of the problems involving the full saturation of the wire, necessary condition to obtain proper working conditions. Bi-metallic wires (magnetic layer on copper wire, carrying the excitation current) have been proposed to achieve full saturation using lower current. In this paper, we present a further improvement: we realized microwires with insulation layer between the copper wire and the magnetic layer. The current flows only into the copper, regardless of the working frequency. Using insulation layer, we achieve $20 \mathrm{~mA}$ saturation current at $10 \mathrm{kHz}$, which is 3 times smaller than for similar wires without insulation layer.

Index Terms-Electrodeposition, insulation layer, magnetic sensors, orthogonal fluxgate, Permalloy.

\section{INTRODUCTION}

$\mathbf{O}$ RTHOGONAL fluxgates are a particular type of orthogonal fluxgate sensor, so called because the excitation field lies in a plane orthogonal to the measured field.

First examples of orthogonal fluxgates had a core composed by a cylinder of soft magnetic material and an excitation coil wound around it. An ac current is injected into the excitation coil in order to generate and excitation field $\mathrm{H}_{\mathrm{ex}}$. If $\mathrm{H}_{\mathrm{ex}}$ is high enough, periodical saturation of the magnetic cylinder occurs in both clockwise and anticlockwise directions.

When a magnetic field $\mathrm{H}_{\mathrm{m}}$ is applied in longitudinal direction (that is parallel to the cylinder), the magnetic domains rotating under the effect of $\mathrm{H}_{\mathrm{ex}}$, will be deflected, resulting in a net component of magnetic flux in longitudinal direction [1]. The variation of longitudinal flux is detected by a pickup coil wound around the core. The amplitude of the voltage induced in the pickup coil will be proportional to the measured field $\mathrm{H}_{\mathrm{m}}$.

Orthogonal fluxgate principle has been forgotten for many years as the mainstream of fluxgate development was on parallel type. Orthogonal fluxgates have been recently rediscovered, due to employment of magnetic microwires as core for such sensors [2].

In this case, the core is composed of a single magnetic microwire: an ac current flows into the wire, generating the circumferential excitation field. In this way, we avoid the necessity to use any excitation coil (a pickup coil will still be necessary to measure the variation of longitudinal field).

Such fluxgates are easier to produce, as one does not need to wind an excitation coil; moreover, they can be manufactured in smaller dimensions.

Unfortunately, it is not possible to fully saturate the whole volume of the magnetic wire. Indeed, assuming that the current flowing into the wire has uniform distribution, the excitation field $\mathrm{H}_{\mathrm{ex}}$ increases linearly from the center of the microwire till its border, as shown in Fig. 3. The inner part of the wire, where $\mathrm{H}_{\mathrm{ex}}$ is lower than saturation field $\mathrm{H}_{\mathrm{s}}$, will not be saturated to cir-

Manuscript received March 06, 2009; revised April 29, 2009. Current version published September 18, 2009. Corresponding author: M. Butta (e-mail: buttam1@fel.cvut.cz).

Digital Object Identifier 10.1109/TMAG.2009.2024885

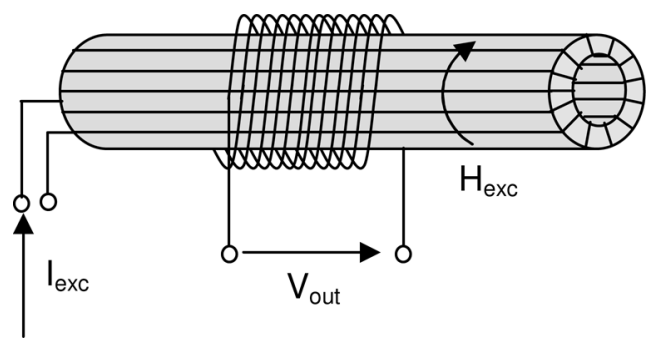

Fig. 1. Classical structure of an orthogonal fluxgate.

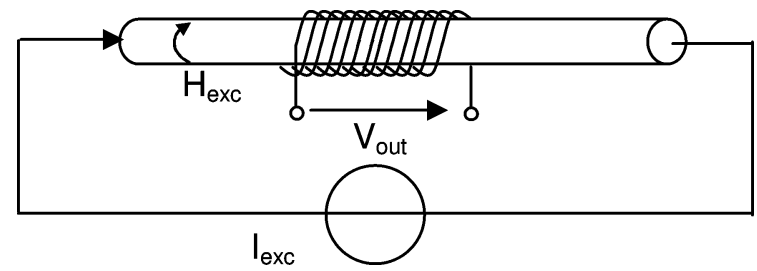

Fig. 2. Orthogonal fluxgate with microwire core.

cumferential direction. When we apply $\mathrm{H}_{\mathrm{m}}$ field comparable or higher than resulting $\mathrm{H}_{\mathrm{ex}}$ in the inner part of the microwire, the magnetization of such inner region could switch to longitudinal direction. Since the excitation field $\mathrm{H}_{\mathrm{ex}}$ is not strong enough; the inner part of magnetic layer will keep such orientation even after removal of in $\mathrm{H}_{\mathrm{m}}$. Therefore, we could have a change of sensor's offset, depending on direction and amplitude of previously applied $\mathrm{H}_{\mathrm{m}}$ (hysteresis in sensor's characteristic).

One could try to reduce this problem by increasing the excitation current: the unsaturated part will be smaller. Nevertheless, the unsaturated part cannot be totally removed: there will always be a small inner part nonsaturated. Moreover, any increment of the excitation current will increase the power consumption: the temperature of the microwire could increase and cause drifts of the sensor offset and change of its sensitivity.

\section{Bi-METALlic WiRES}

In order to overcome this problem, bi-metallic microwires have been proposed. They are composed by a copper core, covered by a layer of magnetic material, usually produced by elec- 


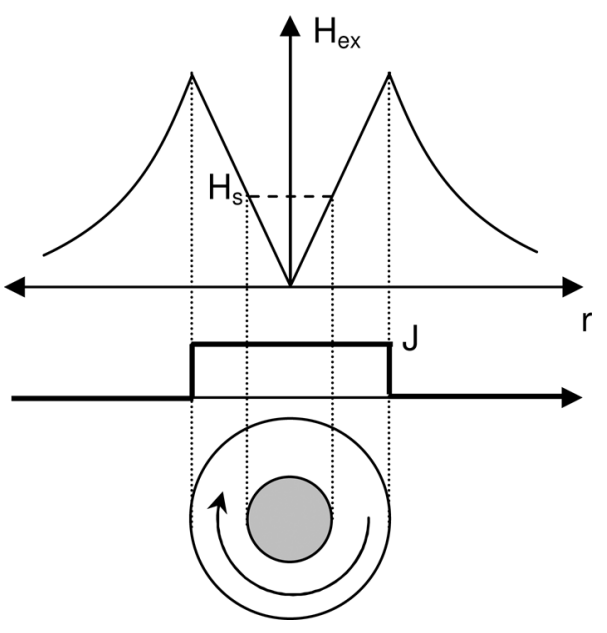

Fig. 3. Excitation field in a magnetic wire with uniform current distribution. The central part of the wire is not saturated.

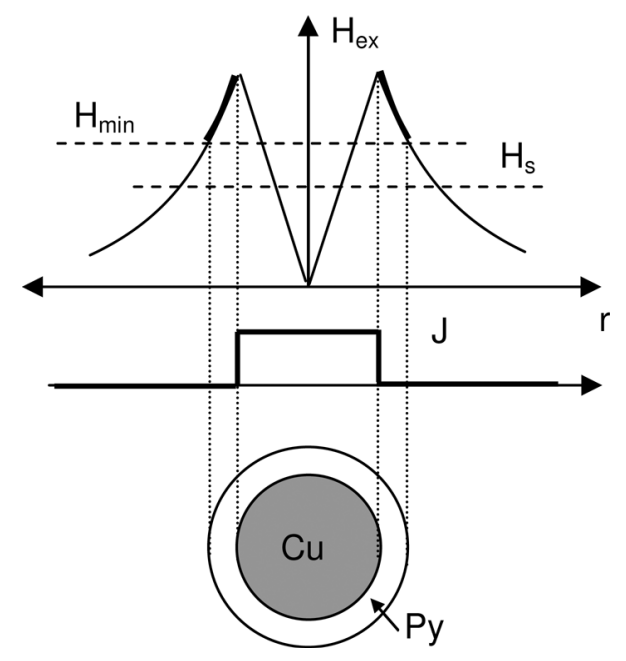

Fig. 4. Excitation field in a bimetallic wire $(\mathrm{Cu} / \mathrm{Py})$, with current flowing only into the copper core. The minimum excitation field $\mathrm{H}_{\min }$ is at the border of the wire. As far as $\mathrm{H}_{\min }$ is higher than coercivity, the wire is fully saturated.

trodeposition [3]. A typical material used for this purpose is Permalloy. Since the resistivity of copper is much lower than resistivity of Permalloy, we assume all the current flowing into the copper core. In this case the excitation field $\mathrm{H}_{\mathrm{ex}}$, increases linearly from the center of the wire till the border between copper and permalloy, then it will decrease as the inverse of the radius [Fig. 4].

In this case the inner part of the permalloy layer will be exposed to the maximum field, whereas the outer part of the layer to the minimum field $\mathrm{H}_{\min }$. If $\mathrm{H}_{\text {min }}$ is higher than $\mathrm{H}_{\mathrm{s}}$ (as shown in Fig. 4), then the whole layer will be completely saturated.

Let us suppose the current does not flow only in the copper core but partially also in the permalloy layer; this is more realistic situation [4]. In this case the inner part of the permalloy will be exposed to the still increasing exciting field [Fig. 5]. Therefore, we would have two minimum values $\mathrm{H}_{\min \_ \text {int }}$ and $\mathrm{H}_{\text {min _ext }}$. In order to achieve full saturation of the microwire, both minimum values of excitation field must be higher than $\mathrm{H}_{\mathrm{s}}$. Depending on the geometry of the microwire, and the working

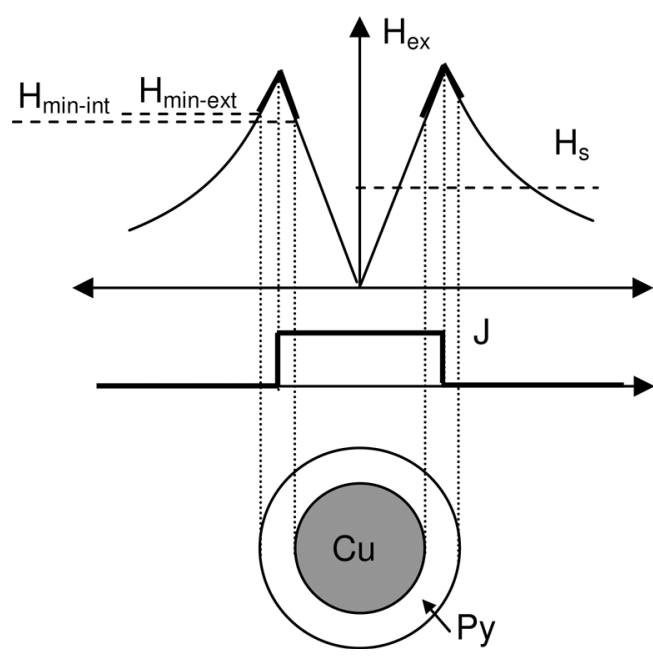

Fig. 5. Excitation field in bimetallic wire with current flowing also into the Permalloy region. The inner part of the magnetic layer could be unsaturated.

frequency of the excitation current, either the external region or the inner shell of the microwire could be unsaturated.

The higher is the frequency, the more the current density will be higher far from the center of the wire. This causes the inner part of the wire to be unsaturated. Any increment of frequency can be useful to achieve higher sensitivity, but the exposed problem is a limit to the choice of working frequency.

Another way to increase sensitivity is to increment the thickness of the magnetic layer. In this case, we cannot increase it too much, to avoid the outer region of the wire to fall under a distance where $\mathrm{H}_{\mathrm{ex}}$ is lower than $\mathrm{H}_{\mathrm{s}}$.

In any case the presence of an unsaturated part in the microwire, requires undesired increment of excitation current, to actually achieve saturation of the whole wire.

\section{INSULATION LAYER}

In order to solve the problem of unsaturated inner region of Permalloy layer, we must try to keep all excitation current flowing through the copper core, in such a way that it does not drain to the magnetic layer. Unfortunately, it is not sufficient to use two materials with very different resistivity: this could work fine for static current, but at higher frequencies (tens of $\mathrm{kHz}$ ), surface effect prevails.

In this case, the only way to keep current into the copper core is the application of an insulation layer between the copper and the magnetic field. Fig. 6 shows the procedure we used to produce a magnetic microwire with such insulation layer.

At first we consider a $4 \mathrm{~cm}$ long, glass-coated copper wire, commercially available (50 $\mu$ m diameter) (1) and we cover its terminations with $\sim 5 \mathrm{~mm}$ long plastic film (2). Then, we applied a few nm gold layer by sputtering (3). Next, we remove the film around the termination and we uncover the nonsputtered portion of wire (4). Once we have the sputtered wire we can perform electrodeposition: we mount the wire on a conductive support, in such a way that the sputtered gold is in contact with the support, and we inject the electrodeposition current. The current will flow from the bath to the gold making the Permalloy growing only along the sputtered area: no Permalloy will be electrodeposited on the termination dipped into the bath (shown on the right side). On the other termination, the Permalloy layer 
1

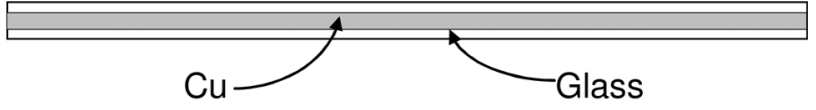

2

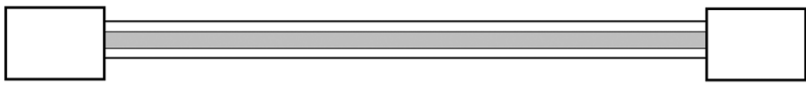

3

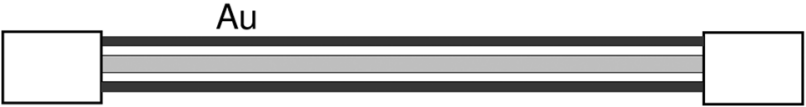

4

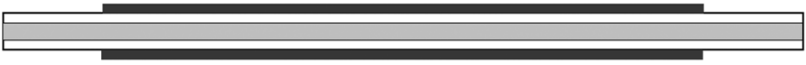

5

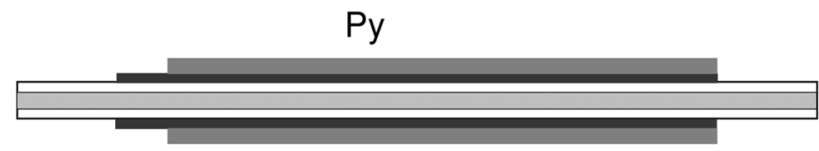

6

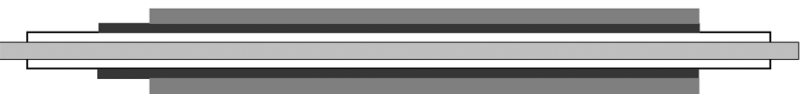

Fig. 6. Procedure for realization of magnetic microwire with insulation layer between the copper core and the magnetic layer (not to scale).

ends at the surface of the bath: therefore there will be still few $\mathrm{mm}$ of sputtered gold without electrodeposited Permalloy, because it stays out of the bath during the electrodeposition (left termination) (5). However, from the magnetic point of view this asymmetry does not cause any problem. Finally, we remove the glass from the termination by microscope assisted scratching, using a very sharp blade (6). Therefore we will have two terminations without insulation, where we can inject excitation current. This current will flow only into the copper core, because it is completely insulated, as well as the terminations, from the gold layer.

More details about electroplating fabrication technique can be found in [5].

\section{RESULTS}

We have produced several microwires using the procedure explained above. All of them have around $58 \mu \mathrm{m}$ total diameter.

We have characterized them as explained in [6]. We found out that the current necessary to achieve saturation for these wires is much lower than for wires with similar geometry, and the same material (Permalloy) composing the magnetic layer, but without any insulation layer [7].

In the worst case the saturation current for wires with insulation current was around $20 \mathrm{~mA}$ at $10 \mathrm{kHz}$. In some other cases it could be as low as $15 \mathrm{~mA}$. Similar wires without insulation layer have saturation current usually around $60 \mathrm{~mA}$, at $10 \mathrm{kHz}$.

This means we achieved a 3 times lower saturation current, with related advantages in terms of power consumption and thermal dilatation.

Fig. 7 shows the dependence of $\mathrm{H}_{c}$ on the working frequency, when we saturate two wires at $90 \mathrm{~mA}$, with and without insulation layer. We have calculated the exciting field (and therefore also the coercivity) from the excitation current, assuming it is flowing only into the copper core.

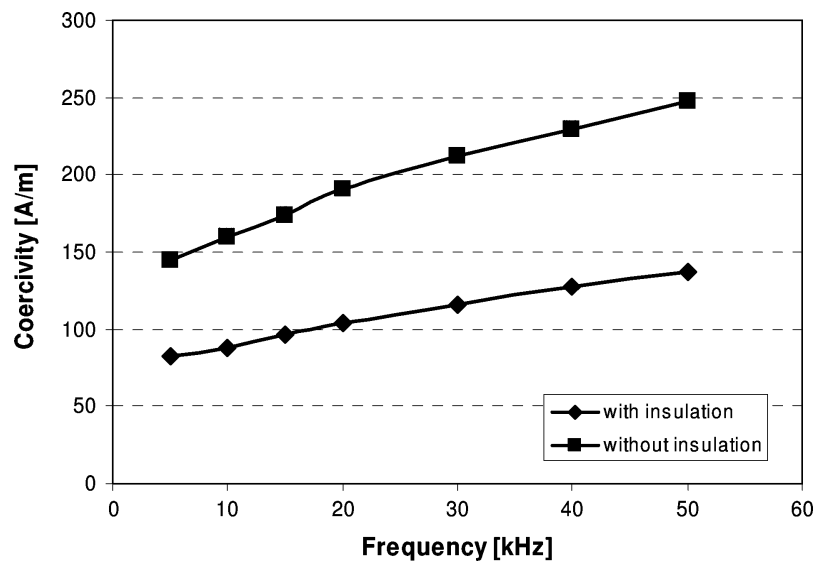

Fig. 7. Dependence of coercivity on frequency of excitation current, in case of microwires with and without insulating layer.

In fact, we can observe that without insulation layer $\mathrm{H}_{\mathrm{s}}$ is much bigger than with insulation layer. This is probably due to the fact that excitation current does not flow only into the copper core: in this case, the real excitation field is actually smaller than the value calculated by assuming current flowing only into the copper core. Increasing the frequency of excitation current, the coercivity increases as expected. However, we should notice that the coercivity increases faster in case of no insulation layer. This is probably given by the change of current density distribution in the wire. This extra contribution is present only in case of no insulation layer: when we use insulation layer we can disregard the distribution of current density in the copper core, because the field generated out of the copper depends only on the total current included into the circumference and not on its distribution.

\section{CONCLUSION}

In this paper, we have presented the issues we must face when we use a magnetic microwire as core for orthogonal fluxgate. Due to surface effect, the excitation current drains from the copper core to the magnetic layer: it results in a lower excitation field in the inner part of the magnetic layer. We propose the employment of a glass layer between the copper core and the magnetic layer, in order to keep the current flowing only into the copper core. We have developed a process which allows us to easily fabricate such microwires.

The resulting wires have much lower saturation current than Copper/Permalloy wires with comparable geometry but without insulation layer. It must be mentioned that we consider as the saturation current the amplitude of the current which fully saturates the magnetic layer, including the inner region. Therefore, when we excite the microwire by using such current we do not observe hysteresis into the characteristic of the fluxgate. In fact, we define the amplitude of the saturation current from the sensor's characteristic. Therefore, the declared values of saturation current include an extra amount of current we should add to overcome the hysteresis of sensor's characteristic, due to unsaturated inner region of Permalloy layer. The much lower saturation current, achieved in realized microwires with glass insulation layer, tells us that also the hysteresis problem is solved without need of additional increment of excitation current. 
The change of offset due to extremely large field (perming) is rather due to change of magnetization in defects spots of Permalloy layer, which are much harder to magnetize than the material itself. In this case the perming effect depends on the quality of the magnetic material; the geometry and the structure of the wire do not affect the perming effect.

\section{ACKNOWLEDGMENT}

This work was supported by the Czech Grant Agency under Grant No. GA102/08/0743 "Fluxgate effect in thin layers."

\section{REFERENCES}

[1] M. Butta and P. Ripka, "Two-domain model for orthogonal fluxgate," presented at the INTERMAG08 Conf., Madrid, May 4-8, 2008.

[2] I. Sasada, J. Appl. Phys., vol. 91, p. 7789, 2002.

[3] J. Fan, X. P. Li, and P. Ripka, J. Appl. Phys., vol. 99, p. 08B311, 2006.

[4] J. P. Sinecker et al., J. Magn. Magn. Mater., vol. 249, pp. 16-21, 2002.

[5] K. Pirota, M. Hernández-Vélez, D. Navas, A. Zhukov, and M. Vázquez, Adv. Funct. Mater., vol. 14, p. 266, 2004.

[6] P. Ripka et al., Sens. Actuators A: Phys., 2007, doi:10.1016/j.sna. 2007.10.008

[7] F. E. Atalay et al., J. Phys. D: Appl. Phys., vol. 39, pp. 431-436, 2006. 